\section{Analysis of Chattering in Continuous Sliding-Mode Controllers}

\author{
I. Boiko and L. Fridman
}

\begin{abstract}
An analysis of two most popular continuous sliding-mode algorithms: The power-fractional sliding-mode algorithm and a second-order sliding-mode algorithm known as the super-twisting is carried out in the frequency domain with the use of the describing function method. It is shown that in the presence of an actuator, the transient process converges to a periodic motion. Parameters of this periodic motion are analyzed. A few examples are considered to illustrate the obtained results.
\end{abstract}

Index Terms-Chattering, relay control, sliding-mode (SM), variable structure systems.

\section{INTRODUCTION}

Continuous sliding-mode (SM) algorithms were developed as a means of mitigating the drawbacks of the discontinuous classical SM such as chattering phenomenon and infinite-time convergence. Two control algorithms that introduce a continuous control law with infinite gain known as the power-fractional SM controller (PFSM), which serves as a basis of the terminal SM concept, and the super-twisting (ST) were proposed in [1]-[3], respectively. ${ }^{1}$ Both utilize a continuous nonlinear function with infinite gain. The popularity of continuous SM algorithms can be illustrated by numerous publications devoted to the theory and applications of this idea [4]-[8].

It is known that the first order SM in systems with actuators of relative degree $t w o$ or more is realized as chattering [9]-[11]. It was proved in [12] that the same property is valid not only for the relay type of SM control but also for other types of first-order discontinuous control algorithms (i.e., linear switched gains, etc.). However, it has been a popular notion over the last decade that the second-order SM control would offer chattering elimination [4]-[6]. Yet in [13], where a popular second-order SM algorithm known as "twisting" was analyzed, it was shown that the second-order SM control also exhibits chattering in the presence of parasitic dynamics. The same "chattering-free" property has been associated with the continuous SM control algorithms. This conclusion was merely made from the continuity property of the control without analyzing the possible causes of chattering: Presence of parasitic dynamics, delays and hysteresis in the nonlinear element. Since the algorithms to be analyzed in the note involve a nonlinear function with infinite gain, which is a potential source of instability, those algorithms are likely to excite oscillations under certain conditions. The aim of this note is to analyze the two aforementioned algorithms, to show

Manuscript received July 26, 2004; revised December 19, 2004 and April 10, 2005. Recommended by Associate Editor M. Reyhanoglu. The work of L. Fridman was supported by the Mexican CONACyT (Consejo Nacional de Ciencia y Tecnología) under Grant 43807-Y, and by the Programa de Apoyo a Proyectos de Investigacion e Innovacion Tecnolgica (PAPIIT) UNAM under Grant 117103.

I. Boiko is with SNC-Lavalin, Calgary, AB T2P 3G5, Canada (e-mail: iboiko@ieee.org).

L. Fridman is with the Facultad de Ingeniería. the Universidad Nacional Autónoma de México, UNAM, Ciudad Universitaria, CP 04510 Mexico City, Mexico (e-mail: 1fridman@verona.fi-p.unam.mx).

Digital Object Identifier 10.1109/TAC.2005.854655

${ }^{1}$ There also exist other control algorithms the most popular of which is a finite gain continuous control in a boundary layer (saturation). However, this approach does not have some typical of the SM control features and for that reason is not considered in this note. that the property of being "chattering-free" associated with the continuous SM algorithms is a misconception, to prove possible existence of periodic motions (chattering) in the systems with actuators driven by those algorithms, and to provide a methodology of assessment of parameters of those motions.

Given the objective of the outlined analysis and the facts that the introduction of an actuator increases the order of the system, and a relatively "inconvenient" nonlinearity is present in the SM algorithm, the analysis of corresponding Poincare maps becomes very complicated. In this case the describing function (DF) method [14] becomes a good choice as a method of analysis, as it provides a relatively simple and efficient solution of the problem.

The note is organized as follows. At first, the model of the system involving the PFSM algorithm is obtained. Then, the DF model of the algorithm suitable for the frequency domain analysis is obtained. It is shown that a periodic motion occurs and the problem of finding the parameters of this periodic motion is considered. After that the same is done for the ST algorithm. Finally, a number of examples are considered.

\section{POWER-Fractional SM AND ITS DF ANALYSIS}

PFSM was proposed in [1], [2] as a continuous SM control algorithm. Let the plant (or plant plus actuator) be given by the following differential equations:

$$
\begin{aligned}
& \dot{\mathbf{x}}=\mathbf{A} \mathbf{x}+\mathbf{B} u \\
& y=\mathbf{C} \mathbf{x}
\end{aligned}
$$

where $\mathbf{A}$ and $\mathbf{B}$ are matrices of respective dimensions, $\mathbf{x} \in \mathbf{R}^{\mathrm{n}}, y \in$ $\mathbf{R}^{1}, y$ can be treated as either the sliding variable or the output of the plant. We assume that the plant is asymptotically stable, apart from some possible integrating terms, and is a low-pass filter. We shall also use the plant description in the form of a transfer function $W(s)$, which can be obtained from the formulas (1) as follows:

$$
W(s)=\mathbf{C}(\mathbf{I} s-\mathbf{A})^{-1} \mathbf{B} .
$$

The control law for PFSM is given as follows:

$$
u(t)=-\beta y^{q / p}
$$

where $\beta>0, q$, and $p$ are positive integers $(p>q), p$ is odd. From (2), one can see that the control is a nonlinear function of the sliding variable $y$. Like in the case of the classical SM control, we assume that the sliding variable is a linear combination of the plant states and, therefore, the plant transfer function from control to sliding variable has relative degree one. The system under analysis can be represented in the form of the block diagram of the closed-loop system as in Fig. 1 (we will assume that input $f(t) \equiv 0$ and, therefore, $y(t)=-\sigma(t)$ ).

Let us apply the DF method [14] to this closed-loop nonlinear system and find under which conditions a periodic motion may exist in this loop. Find the DF of the nonlinearity (2) as the first harmonic of the periodic control signal divided by the amplitude of $y(t)$-in accordance with the definition of the DF.

$$
N=\frac{\omega}{\pi a} \int_{0}^{2 \pi / \omega} u(t) \sin \omega t d t+j \frac{\omega}{\pi a} \int_{0}^{2 \pi / \omega} u(t) \cos \omega t d t
$$

where $a$ is the amplitude of the input to the nonlinearity and $\omega$ is the frequency of $y(t)$ (the output is assumed to be $y(t)=a \sin \omega t$ ). Applying this formula to the nonlinearity given by (2) we can derive the following formula of the DF of PFSM:

$$
N=\frac{2 \beta a^{\frac{q}{p}-1}}{\pi} \int_{0}^{\pi}(\sin \psi)^{\frac{q}{p}+1} d \psi=\frac{2 \beta a^{\frac{q}{p}-1} \Gamma\left(\frac{q}{2 p}+1\right)}{\sqrt{\pi} \Gamma\left(\frac{q}{2 p}+1.5\right)}
$$




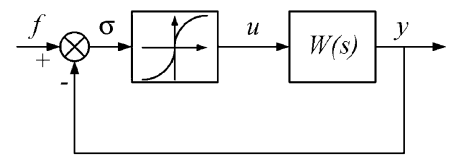

Fig. 1. Block diagram of the system with PFSM.

where $\Gamma$ is gamma-function [15]. The minus sign of (2) is attributed to the negative feedback and not accounted for in formula (3). The integral (3) can be evaluated numerically. For example, if $q=1$ and $p=3$ the formula of the DF can be written as follows:

$$
N \approx 1.159 \beta a^{-2 / 3} \text {. }
$$

One can see from (3) and (4) that $N(a)$ is a real function. For that reason, the negative reciprocal of the DF coincides with the negative part of the real axis on the complex plane. A periodic solution can be found as a point of intersection of the Nyquist plot of the actuatorplant-sliding surface combination and of the negative reciprocal DF [of (3) or (4)], which corresponds to the solution of the harmonic balance equation [14]

$$
W(j \Omega) \cdot N(a)=-1
$$

where $\Omega$ is a frequency of the required periodic solution.

The frequency corresponding to this point would, obviously, be the same as in the relay type of the SM control, as the negative reciprocal of the DF for the ideal relay also coincides with the negative part of the real axis. However, the amplitudes of the oscillations would be different in those two cases. Thus, a periodic motion may occur in the system with PFSM if the combined relative degree of the actuator, plant and sliding surface is three and higher, as in this case the Nyquist plot of the linear part intersects the negative part of the real axis. Stability of this periodic motion can easily be verified via the use of Loeb criterion [14], [16]. For this type of nonlinearity, a stable periodic solution would correspond to the Nyquist plot of the plant intersecting the real axis from below.

Let us compare the amplitudes of possible periodic motions in the system with PFSM and in the system with the relay nonlinearity. For a steady periodic motion, we can write the condition of the balance of the amplitudes as follows:

$$
|W(j \Omega)| \cdot N(a)=1 .
$$

This equation applies to both: The relay control and the PFSM. Since the frequency of the periodic solution $\Omega$ is the same in both cases the value of the DF should be the same for the relay control and the PFSM control. Taking into account that the DF for the relay control is given by the following formula: $N_{R}=4 c /(\pi a)$, where $c$ is the amplitude of the relay, the amplitudes of the oscillations in the system with the relay control and the PFSM are related as follows (for $q=1$ and $p=3$ ):

$$
\frac{4 c}{\pi a_{R}}=1.159 \beta a_{\mathrm{PFSM}}^{-2 / 3}
$$

where $a_{R}$ is the amplitude of $y(t)$ in a periodic motion for the relay control, $a_{\mathrm{PFSM}}$ is the amplitude for the PFSM. Assuming that $c=$ $1, \beta=1$, from (6), we can obtain the following expression:

$$
a_{R}=1.099 a_{\mathrm{PFSM}}^{2 / 3} .
$$

One can see from (7) that the amplitude of a periodic motion (chattering) in the PFSM can be higher or lower than the corresponding amplitude in the relay control-depending on the value of the magnitude of the frequency response $|W(j \Omega)|$ of the linear part at the frequency corresponding to the phase response $-180^{\circ}$. However, for small amplitudes of chattering (if inequality $|W(j \Omega)| \ll 1$ holds) it follows

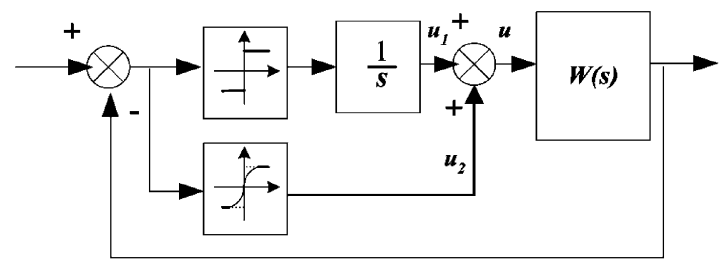

Fig. 2. Block diagram of the system with the super-twisting algorithm.

from (7) that the amplitude of chattering for PFSM is smaller than the one for the relay control.

\section{SUPER-Twisting Algorithm AND ITs DF ANALYSIS}

\section{A. DF of Super-Twisting Algorithm}

The super-twisting algorithm is one of the popular algorithms among the second order sliding-mode algorithms. It is used for the plants of relative degree one. The control $u$ for the super-twisting algorithm is given as a sum of two components [3]

$$
\begin{aligned}
u(t) & =u_{1}(t)+u_{2}(t) \\
\dot{u}_{1} & =-\gamma \operatorname{sign}(y) \\
u_{2} & =\left\{\begin{array}{lll}
-\lambda\left|s_{0}\right|^{\rho} \operatorname{sign}(y), & \text { if } & |y|>s_{0} \\
-\lambda|y|^{\rho} \operatorname{sign}(y), & \text { if } & |y| \leq s_{0}
\end{array}\right.
\end{aligned}
$$

where $\gamma, \rho$, and $s_{0}$ are design parameters, $0.5 \leq \rho<1$.

The system under analysis can be represented in the form of the block diagram as in Fig. 2.

With the square root nonlinearity $(\rho=0.5)$ the DF formula can be derived as

$$
\begin{aligned}
N_{2} & =\frac{2}{\pi a_{y}} \int_{0}^{\pi} \lambda \sqrt{a_{y} \sin \psi} \sin \psi d \psi \\
& =\frac{2 \lambda \sqrt{a_{y}}}{a_{y} \sqrt{\pi}} \frac{\Gamma(1.25)}{\Gamma(1.75)} \approx \frac{1.1128 \lambda}{\sqrt{a_{y}}}
\end{aligned}
$$

where $a_{y}$ is the amplitude of variable $y, a_{y} \leq s_{0}$ (that is considered the most important range of the amplitude values for the analysis of the steady state) and $\Gamma$ is the gamma-function.

For an arbitrary value of the power $\rho$ in (8) and the amplitude $a_{y} \leq$ $s_{0}$, the formula of the DF of such nonlinear function can be given as follows:

$$
\begin{aligned}
N_{2} & =\frac{2 \lambda a_{y}^{\rho-1}}{\pi} \int_{0}^{\pi}(\sin \psi)^{\rho+1} d \psi \\
& =\frac{2 \lambda a_{y}^{\rho-1}}{\sqrt{\pi}} \frac{\Gamma\left(\frac{\rho}{2}+1\right)}{\Gamma\left(\frac{\rho}{2}+1.5\right)}, \quad 0<\rho<1 .
\end{aligned}
$$

The DF of the first component of the super-twisting algorithm can be written as follows:

$$
N_{1}=\frac{4 \gamma}{\pi a_{y}} \frac{1}{j \omega}
$$

which is a result of the cascade connection of the ideal relay with the DF equal to $4 \gamma /\left(\pi a_{y}\right)$ [14] and the integrator with the transfer function $1 / s$ (for the harmonic signal the Laplace variable $s$ can be replaced with $j \omega)$. With the account of both control components, the DF of the super-twisting algorithm can be written as

$$
N=N_{1}+N_{2}=\frac{4 \gamma}{\pi a_{y}} \frac{1}{j \omega}+1.1128 \frac{\lambda}{\sqrt{a_{y}}} .
$$

Let us note that the DF of the super-twisting algorithm depends on both: The amplitude and the frequency values. The parameters of the limit cycle can be found via the solution of the complex equation (5), 
where the DF $N$ is given by (11). The function at the negative reciprocal of the DF can be represented by the following formula:

$$
\begin{aligned}
-\frac{1}{N} & =-\frac{1}{1.1128 \frac{\lambda}{\sqrt{a_{y}}}+\frac{4 \gamma}{\pi a_{y}} \frac{1}{j \omega}} \\
& =-\frac{0.8986 \frac{\sqrt{a_{y}}}{\lambda}+j 1.1329 \frac{\gamma}{\lambda^{2}} \frac{1}{\omega}}{1+1.3092 \frac{\gamma^{2}}{\lambda^{2}} \frac{1}{a_{y} \omega^{2}}} .
\end{aligned}
$$

The function $-1 / N$ is a function of two variables: The amplitude and the frequency. It can be depicted as a number of plots representing the amplitude dependence, with each of those plots corresponding to a certain frequency. The frequency range that is of interest lies below the frequency corresponding to the intersection of the Nyquist plot and the real axis. The plots of function $-1 / N$ are depicted in Fig. 3. The plots $1-4$ correspond to four different frequencies, with the following relationship: $\omega_{1}>\omega_{2}>\omega_{3}>\omega_{4}$. Each of those plots represents the dependence of the DF on the amplitude value.

Function $-N^{-1}\left(a_{y}\right)$ (where $\omega=$ const) has an asymptote at $a_{y} \rightarrow$ $\infty$, which is the horizontal line $-j 1.1329 \gamma /\left(\lambda^{2} \omega\right)$. Also, it is easy to show that $\lim _{a_{y} \rightarrow 0} \arg \left(-N^{-1}\left(a_{y}\right)\right)=-\pi / 2$.

\section{B. Existence of the Periodic Solutions}

The solution of the harmonic balance (5) can be iterative with possible application of various techniques. However, complex (5) with two unknown variables: $a_{y}$ and $\Omega$ can be reduced to one real equation having only one unknown variable $\Omega$ as follows. Write (5) in the form of $N\left(a_{y}\right)=-W^{-1}(j \Omega)$, where $N\left(a_{y}\right)$ is given by (11)

$$
\frac{4 \gamma}{\pi a_{y}} \frac{1}{j \Omega}+1.1128 \frac{\lambda}{\sqrt{a_{y}}}=-W^{-1}(j \Omega) .
$$

Considering the real part of both sides, we can obtain

$$
\frac{1.1128 \lambda}{\sqrt{a_{y}}}=-\operatorname{Re} W^{-1}(j \Omega)
$$

Express $a_{y}$ from the aforementioned equation and substitute this value in the equation, which can be obtained by considering the imaginary parts of the previous complex equation. Finally, one equation with one unknown variable $\Omega$ can be obtained as follows:

$$
\Psi(\Omega)=\frac{4 \gamma}{\pi \Omega} \frac{1}{\operatorname{Im} W^{-1}(j \Omega)}-\left(\frac{1.1128 \lambda}{\operatorname{Re} W^{-1}(j \Omega)}\right)^{2}=0 .
$$

Once (13) has been solved, the amplitude $a_{y}$ can be computed as follows:

$$
a_{y}=\frac{4 \gamma}{\pi \Omega} \frac{1}{\operatorname{Im} W^{-1}(j \Omega)} .
$$

Therefore, if a periodic motion occurs its parameters can be found from (13) and (14). Existence of a periodic solution can be proven via analysis of function (13).

Proposition 1: If relative degree of the plant is two or higher and the plant does not have double zero poles there always exists at least one periodic solution of the system with the super-twisting algorithm.

Proof: At first prove the Proposition 1 for the plant of relative degree 3 and higher. Let the plant be dynamics of relative degree three or higher. It follows from the formula of the DF of the algorithm (11) that the periodic solution should always be looked for within the frequency range that corresponds to $-\pi / 2$ and $-\pi$ of the phase characteristic of the plant (see also Fig. 3). Denote frequency $\Omega_{1}$ being the frequency at which the phase characteristic of the plant is $-\pi / 2: \arg W\left(j \Omega_{1}\right)=$ $-\pi / 2$. Similarly denote frequency $\Omega_{2}$ being the frequency at which the phase characteristic of the plant is $-\pi: \arg W\left(j \Omega_{2}\right)=-\pi$. Both frequencies are finite. Find the following two limits of function

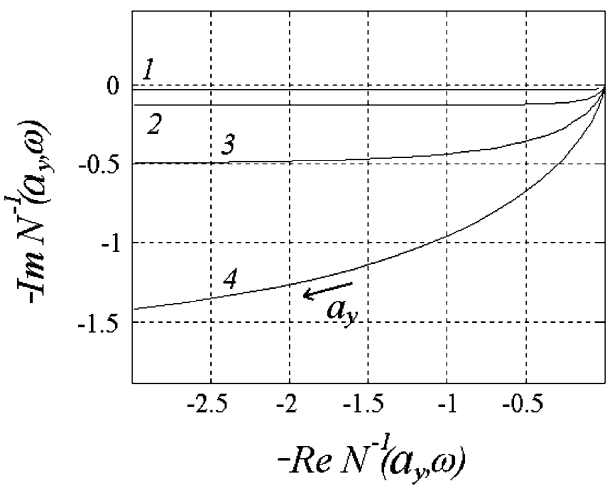

Fig. 3. Plots of function $-1 / N ; \omega_{1}>\omega_{2}>\omega_{3}>\omega_{4}$.

$\Psi(\omega)(13): \Psi\left(\Omega_{1}+\right)=-\infty$ and $\Psi\left(\Omega_{2}-\right)=\infty$. The signs are different. Function $\Psi(\omega)$ is continuous within the range $\omega \in\left[\Omega_{1} ; \Omega_{2}\right]$ that follows from (13). Therefore, within the specified range, there is at least one solution of (13).

Assume now that relative degree of the plant is 2 . In this case, we can define frequency $\Omega_{1}$ in the same manner as before but frequency $\Omega_{2}$ becomes infinite. Let us write the asymptotical representation of the plant transfer function for high-frequency inputs in the polynomial form:

$$
W(s) \approx \frac{1}{a_{2} s^{2}+a_{1} s+a_{0}} .
$$

Then, substituting $j \omega$ for $s$ we have $W^{-1}(j \omega) \approx-a_{2} \omega^{2}+a_{0}+j a_{1} \omega$ and it is easy to see that for sufficiently large $\omega$

$$
\Psi(\omega)=\frac{4 \gamma}{\pi} \frac{1}{a_{1} \omega^{2}}-\frac{1.1128^{2} \lambda^{2}}{\left(a_{0}-a_{2} \omega^{2}\right)^{2}}>0 .
$$

Therefore, between $\Omega_{1}$ and $\Omega_{2}$ there always exists a certain frequency $\Omega$, which provides a solution to the system (1), (8). This completes the proof.

Remark: It is important that the point of the intersection is located in the third quadrant of the complex plane. Therefore, if the transfer function of the plant (or plant plus actuator) has relative degree higher than one a periodic motion may occur in such a system. For that reason, if an actuator of first or higher order is added to the plant of relative degree one driven by the super-twisting controller a chattering (periodic motion) may occur in the system. From Fig. 3 , it also follows that the frequency of the periodic solution for the super-twisting algorithm is always lower than the frequency of the periodic motion in the system with the classical first order SM relay controller, because the latter is determined by the point of intersection of the Nyquist plot and the real axis.

\section{Stability of the Periodic Solution}

Proposition 2: If the following inequality holds:

$$
\operatorname{Re} \frac{h_{1}}{h_{2}+\left.N \frac{\partial \ln W(s)}{\partial s}\right|_{s=j \Omega}}<0
$$

where

$$
h_{1}=\frac{1.1128 \lambda}{2 a_{y} \sqrt{a_{y}}}-j \frac{4 \gamma}{\pi} \frac{1}{\Omega a_{y}^{2}} \quad h_{2}=\frac{4 \gamma}{\pi} \frac{1}{\Omega^{2} a_{y}}
$$

then the periodic solution given by formula (13) is locally orbitaly asymptotically stable.

Proof: To investigate the stability of the solution to the system (1), (8), we consider the system transients due to small perturbations 
of this solution when $a_{y}$ is quasistatically varied to $\left(a_{y}+\Delta a\right)$. As in the proof of Loeb criterion [14], [16], we assume that the harmonic balance equation still holds for slight perturbations, so that a damped oscillation of the complex frequency $j \Omega+(\Delta \sigma+j \Delta \Omega)$ corresponds to the modified amplitude $\left(a_{y}+\Delta a\right)$

$$
N\left(a_{y}+\Delta a, j \Omega+(\Delta \sigma+j \Delta \Omega)\right) \cdot W(j \Omega+(\Delta \sigma+j \Delta \Omega))=-1
$$

where the DF $N\left(a_{y}, \Omega\right)$ is given by formula (11). The nominal solution is determined by zero perturbations: $\Delta \sigma=\Delta \Omega=\Delta a=0$. Considering the variations around the nominal solution defined by $\Omega$ and $a_{y}$, following the proof of the Loeb criterion for the stability of the periodic solution let us find the conditions when $\Lambda=\Delta \sigma / \Delta a$ is negative. Write an equation for the amplitude perturbation $\Delta a$. For that purpose take the derivative of (16) with respect to $\Delta a$ as follows:

$$
\begin{aligned}
& \left\{\left.\frac{d N(\Delta a, \Delta \sigma, \Delta \Omega)}{d \Delta a}\right|_{\Delta a=0} \cdot W(j \Omega)\right. \\
& \left.\quad+\left.\frac{d W(\Delta \sigma, \Delta \Omega)}{d \Delta a}\right|_{\Delta a=0} \cdot N\left(a_{y}, \Omega\right)\right\} \Delta a=0 .
\end{aligned}
$$

Take derivatives of $N$ and $W$ considering them composite functions

$$
\begin{aligned}
\left.\frac{d N}{d \Delta a}\right|_{\Delta a=0}= & j \frac{4 \gamma}{\pi} \frac{\Omega}{a_{y}^{2}}-\frac{1.1128 \lambda}{2 a_{y} \sqrt{a_{y}}} \\
& +\frac{4 \gamma}{\pi} \frac{a_{y}}{\Omega^{2}}\left(\frac{d \Delta \sigma}{d \Delta a}+j \frac{d \Delta \Omega}{d \Delta a}\right) \\
\left.\frac{d W}{d \Delta a}\right|_{\Delta a=0}= & \left.\frac{d W}{\mathrm{ds}}\right|_{s=j \Omega}\left(\frac{d \Delta \sigma}{d \Delta a}+j \frac{d \Delta \Omega}{d \Delta a}\right) .
\end{aligned}
$$

Solving (17) for $((d \Delta \sigma) /(d \Delta a)+j(d \Delta \Omega) /(d \Delta a))$ with account of (18) and (19) we can obtain an analytical formula. Considering only the real part of this formula, we obtain (15). This completes the proof.

\section{EXAMPLES OF ANALYSIS AND COMPARISON OF RESULTS}

Example 1: Let the plant be given by the following equations:

$$
\begin{aligned}
\dot{x}_{1} & =x_{2} \\
\dot{x}_{2} & =-x_{1}-x_{2}+u_{a} \\
y & =x_{1}+x_{2}
\end{aligned}
$$

and the actuator by: $0.01 \dot{u}_{a}+u_{a}=u$. Carry out analysis of periodic motions in the systems with the super-twisting controller if the parameters of the algorithm are given as: $\rho=0.5, \gamma=0.8, \lambda=0.6$. The transfer function $W(s)$ of the actuator-plant can be derived from the original equations as

$$
W(s)=\frac{1}{0.01 s+1} \frac{s+1}{s^{2}+s+1} .
$$

Equation (13) has a solution: $\Omega=66.16 \mathrm{~s}^{-1}$. The amplitude $a_{y}$ can be computed with the use of formula (14): $a_{y}=2.33 \cdot 10^{-4}$. Now let us check the stability condition of this periodic solution. The value of $(d \Delta \sigma) /(d \Delta a)$ found as per (15) is $-3.267 \cdot 10^{4}$. Therefore, the periodic solution is locally orbitaly asymptotically stable and corresponding periodic motion exists in the system.

The graphical illustration of the application of formula (5) to the analysis of the periodic motion is presented in Fig. 4. The plot $-N^{-1}\left(a_{y}\right)$ is drawn for the frequency of the periodic motion $\omega=66.16 \mathrm{~s}^{-1}$ obtained previously.

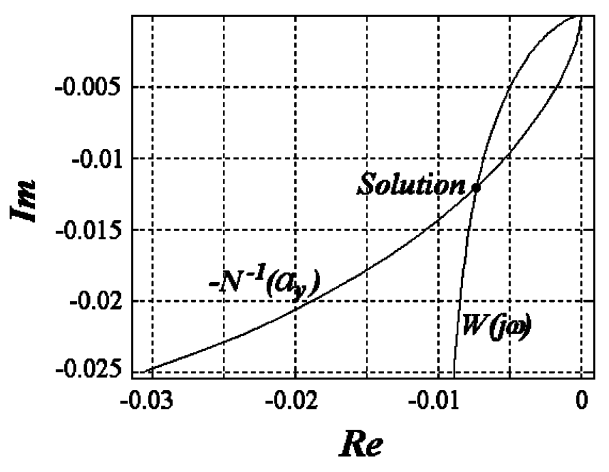

Fig. 4. Negative reciprocal of DF $-N^{-1}\left(a_{y}\right)$ and the Nyquist plot $W(j \omega)$.

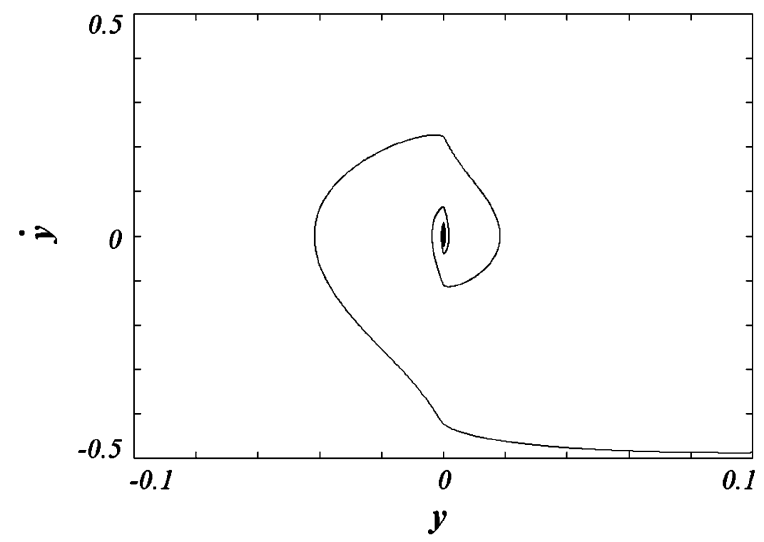

Fig. 5. Super-twisting trajectory.

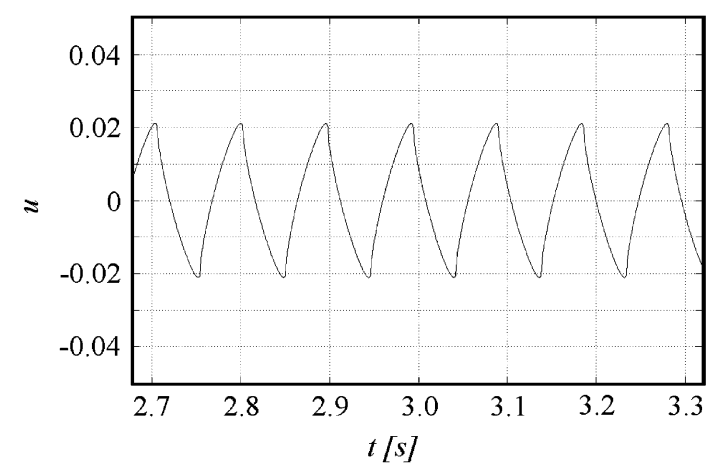

Fig. 6. Control $\mathbf{u}(\mathrm{t})$ at $\mathrm{t} \rightarrow \infty$.

It is clearly seen in the Fig. 4 that the point of intersection exists and the asymptotic behavior of the functions is in accordance with the above analysis. The simulations of the original equations of the system with the super-twisting algorithm produce the following trajectory (Fig. 5).

Fig. 5 displays the trajectory typical of the super-twisting algorithm. The periodicity of the steady state motion is clearly seen in Fig. 6 where the control is presented as a function of time.

The frequency of the periodic motion obtained as a result of the simulation is $\Omega_{\mathrm{sim}}=64.96 \mathrm{~s}^{-1}$, which matches very well the result of the DF analysis.

Some other examples of analysis are presented in Table I. The actuator transfer function is denoted as $W_{a}(s)$, the plant transfer function as $W_{p}(s)$, and the transfer function from the plant input to the sliding 
TABLE I

RESULTS OF COMPUTING AND SiMULLATIONS

\begin{tabular}{|c|c|c|c|c|c|c|}
\hline & $\begin{array}{l}\text { Super- } \\
\text { twisting } \\
\text { controller } \\
(\rho=0.5)\end{array}$ & $\begin{array}{l}\text { Super- } \\
\text { twisting } \\
\text { controller } \\
(\rho=0.5)\end{array}$ & $\begin{array}{l}\text { First order } \\
\text { SM } \\
\text { controller }\end{array}$ & $\begin{array}{l}\text { First order } \\
\text { SM } \\
\text { controller }\end{array}$ & $\begin{array}{c}\text { PFSM } \\
(q / p=0.333)\end{array}$ & $\begin{array}{c}\text { PFSM } \\
(q / p=0.333)\end{array}$ \\
\hline \multirow[t]{2}{*}{ Plant $W_{p}(s)$} & $s+1$ & $s+1$ & $s+1$ & $s+1$ & $s+1$ & $s+1$ \\
\hline & $\overline{s^{2}+s+1}$ & $\overline{s^{2}+s+1}$ & $\overline{s^{2}+s+1}$ & $\overline{s^{2}+s+1}$ & $\overline{s^{2}+s+1}$ & $\overline{s^{2}+s+1}$ \\
\hline Actuator & 1 & 1 & 1 & 1 & 1 & 1 \\
\hline$W_{a}(s)$ & $\overline{0.01 s+1}$ & $0.0001 s^{2}+0.01 s+1$ & $\overline{0.01 s+1}$ & $0.0001 s^{2}+0.01 s+1$ & $\overline{0.01 s+1}$ & $0.0001 s^{2}+0.01 s+1$ \\
\hline$W(s)$ & $W_{p} W_{a}$ & $W_{p} W_{a}$ & $W_{p} W_{a}$ & $W_{p} W_{a}$ & $W_{p} W_{a}$ & $W_{p} W_{a}$ \\
\hline $\begin{array}{l}\Omega(\mathrm{DF} \\
\text { analysis) }\end{array}$ & 66.16 & 55.18 & Infinite & 100.00 & Infinite & 100.00 \\
\hline $\begin{array}{l}\Omega \\
\text { (simu- } \\
\text { lations) }\end{array}$ & 64.96 & 54.14 & $\begin{array}{l}\text { Converging } \\
\text { to infinity }\end{array}$ & 99.26 & $\begin{array}{l}\text { Converging } \\
\text { to infinity }\end{array}$ & 99.67 \\
\hline $\begin{array}{l}\text { Amplitudes } \\
\text { of } \\
\text { chattering } \\
\text { of plant } \\
\text { output }\end{array}$ & $2.33 \mathrm{e}-4$ & $4.81 \mathrm{e}-4$ & 0 & $1.27 \mathrm{e}-4$ & 0 & 1.24 e- 6 \\
\hline
\end{tabular}

variable is denoted as $W_{\sigma}(s)$. As a result, the transfer function of the linear part $W(s)$ is the product of $W_{a}(s)$ and $W_{\sigma}(s)$.

One can see that the results of the DF analysis very well match the results of the simulations. Also, the following properties of the ST algorithm are observed. A periodic motion occurs if the combined relative degree of the actuator and of the plant is higher than one. The frequency of the periodic motion in a system driven by the ST controller is lower than the frequency of the periodic motion in the classical first order relay control- the fact that was predicted by the above analysis. The amplitudes of the chattering reflect the relationship between the frequency of the periodic motion and the decreasing character of the amplitude frequency response $|W(j \omega)|$ of the actuator-plant.

The reader can compare the parameters of chattering that occur in the systems with the same actuators and plants driven by continuous and discontinuous control algorithms using Table I. and the results of [13].

\section{CONCLUSION}

Two most popular continuous sliding-mode algorithms: The power-fractional SM and a second-order SM algorithm known as super-twisting are analyzed in the frequency domain with the use of the describing function method. It is shown that if the combined relative degree of the actuator and plant is higher than two (for the power-fractional SM) or one (for the super-twisting) a periodic motion may occur in the system. Algorithms of finding the parameters of the periodic motions are presented. Some examples of analysis are presented.

\section{REFERENCES}

[1] Z. Man, A. P. Poplinsky, and H. R. Wu, "A robust terminal sliding-mode control scheme for rigid robot manipulators," IEEE Trans. Autom. Control, vol. 39, no. 12, pp. 2465-2469, Dec. 1994.

[2] T. Yu, "Terminal sliding-mode control for rigid robots," Automatica, vol. 34, no. 1, pp. 51-56, 1998.

[3] A. Levant, "Sliding order and sliding accuracy in sliding-mode control," Int. J. Control, vol. 58, pp. 1247-1263, 1993.

[4] G. Bartolini, A. Ferrara, and E. Usai, "Chattering avoidance by secondorder sliding-mode control," IEEE Trans. Autom. Control, vol. 43, no. 2, pp. 241-246, Feb. 1998.
[5] G. Bartolini, A. Ferrara, A. Levant, and E. Usai, "On second order sliding-mode controllers," in Variable Structure Systems, Sliding Mode and Nonlinear. ser. Lecture Notes in Control and Information Science 247, K. D. Young and U. Ozguner, Eds. London, U.K.: Springer-Verlag, 1999, pp. 329-350.

[6] Y. B. Shtessel, I. A. Shkolnikov, and M. J. Brown, "An asymptotic second-order smooth sliding-mode control," Asian J. Control, vol. 5, no. 4, pp. 498-504, 2003.

[7] H. Sira-Ramirez, "Dynamic second-order sliding-mode control of the hovercraft vessel," IEEE Trans. Control Syst. Technol., vol. 10, no. 6, pp. 860-865, Jun. 2002.

[8] J. Alvarez, Y. Orlov, and L. Acho, "An invariance principle for discontinuous dynamical systems with application to a coulomb friction oscillator," J. Dyna. Syst. Measure. Control, vol. 122, no. 4, pp. 687-690, 2000.

[9] Ya. Z. Tsypkin, Relay Control Systems. Cambridge, U.K.: Cambridge Univ. Press, 1985

[10] L. Fridman, "An averaging approach to chattering," IEEE Trans. Autom. Control, vol. 46, no. 8, pp. 1260-1264, Aug. 2001.

[11] _ - "Singularly perturbed analysis of chattering in relay control systems," IEEE Trans. Autom. Control, vol. 47, no. 12, pp. 2079-2084, Dec. 2002.

[12] I. Boiko, "Frequency domain analysis of fast and slow motions in sliding modes," Asian J. Control, vol. 5, no. 4, pp. 445-453, 2003.

[13] I. Boiko, L. Fridman, and I. M. Castellanos, "Analysis of second-order sliding-mode algorithms in the frequency domain," IEEE Trans. Autom. Control, vol. 49, no. 6, pp. 946-950, Jun. 2004.

[14] D. P. Atherton, Nonlinear Control Engineering-Describing Function Analysis and Design. Workingham, U.K.: Van Nostrand, 1975.

[15] Handbook of Mathematical Tables and Formulas, R. S. Burlington, Ed., McGraw-Hill, New York, 1973.

[16] A. Gelb and W. E. Vander Velde, Multiple-Input Describing Functions and Nonlinear System Design. New York: McGraw-Hill, 1968. 